\title{
Demandas fisiológicas y psicológicas en el fútbol*
}

\author{
Henry Humberto León Ariza", Alveiro Sánchez Jiménez"*, \\ Jhon Freddy Ramírez Villada"**t
}

Recibido: 7 de julio de 2011 Revisado: 16 de septiembre de 2011 Aceptado: 4 de octubre de 2011

\section{Resumen}

El fútbol es el deporte más popular del mundo, lo practican hombres, mujeres y niños, en todas las edades y a todo nivel competitivo. En él es necesario un gran conjunto de cualidades físicas, técnicas, tácticas y psicológicas, ajustadas a las demandas del compromiso deportivo.

El objetivo de esta revisión es proveer una visión general de los requerimientos fisiológicos y psicológicos del juego de acuerdo con la más reciente información científica al respecto y con especial énfasis en los protocolos de mayor actualidad aplicados para la evaluación de la resistencia, la fuerza y las expresiones emocionales en el proceso denominado "diagnóstico y control del entrenamiento".

Palabras clave: rendimiento deportivo, condición fisiológica, condición física, técnica, táctica

\footnotetext{
* Artículo de revisión, base de la investigación: "Caracterización fisiológica y psicológica de los futbolistas universitarios que entrenan y compiten a 2600 metros sobre el nivel del mar" (mayo de 2011 - diciembre de 2011).

** Licenciado en Educación Física, Médico Cirujano, docente investigador de la Facultad de Cultura Física, Deporte y Recreación, director de la línea de investigación en fisiología, Universidad Santo Tomás. Correo electrónico: henryleon@usantotomas.edu.co

*** Licenciado en Educación Física, magíster en Desarrollo personal y recursos mentales de las actividades físicas y deportivas, docente investigador de la Facultad de Cultura Física, Deporte y Recreación, director de la línea de investigación en psicología deportiva, Universidad Santo Tomás. Correo electrónico: alveirosanchez@usantotomas.edu.co

**** Licenciado en Educación Física, doctor en Ciencias Aplicadas a la Actividad Física y el Deporte, docente investigador de la Facultad de Cultura Física, Deporte y Recreación, director del Grupo de investigación en ciencias aplicadas al ejercicio físico, el deporte y la salud (GICAEDS), Universidad Santo Tomás. Correo electrónico: jhonramirez@usantotomas.edu.co
} 


\section{Physiological and psychological demands in soccer}

\section{Abstract}

Soccer is the most popular game around the world men, women and children of all ages and levels of competition practice it. For this game a group of physical, technical, tactical and psychological qualities are necessary to confront its demands.

The aim of this review is to provide an overview of the physiological and psychological requirements of the game according to the latest scientific information on the topic with special emphasis in the new protocols applied to recognize the resistance, endurance and emotional expressions in the process named "training diagnosis and control".

Keywords: Performance, psychological condition, physical condition, technic, tactics

\section{Introducción}

El fútbol es considerado por muchos el deporte más popular del mundo y sus practicantes deben reunir un conjunto amplio de habilidades físicas, técnicas y tácticas para su adecuado desempeño, al igual que el desarrollo de un apropiado control mental que les permita afrontar sus demandas psicológicas.

Este tipo de deportes incorporan periodos de alta intensidad física y psicológica que constantemente se combinan con otros de baja intensidad. Dentro de las demandas fisiológicas requeridas se encuentran, entre otras, la resistencia, la fuerza muscular, la flexibilidad y la agilidad (Svensson y Drust, 2005); dichas demandas presentan marcadas diferencias de acuerdo con la posición de juego, con el grado de fatiga, con el estilo de juego y con las condiciones medioambientales 
(Reilly y Gilbourne, 2003). Desde el punto de vista psicológico, el adecuado manejo de la presión psicológica, el control del estrés, la ansiedad y el mantenimiento de la motivación y la concentración son fundamentales para la obtención de logros deportivos (Núñez, 2011).

Tanto para el futbolista como para el entrenador es importante obtener la información que permita clarificar los objetivos del entrenamiento, a la vez que provea de retroalimentación y motivación al jugador (Metaxas, Koutlianos, Sendelides y Mandroukas, 2009).

Este documento presenta los hallazgos más recientes publicados acerca de las demandas fisiológicas y psicológicas a las que se enfrenta un futbolista profesional o aficionado, lo cual a su vez hace parte del marco conceptual de la investigación sobre el fútbol universitario que adelanta la Facultad de Cultura Física, Deporte y Recreación a través de la línea de investigación en Fisiología del Grupo de investigación en ciencias aplicadas al ejercicio físico, el deporte y la salud (GICAEDS).

\section{Demandas fisiológicas en el fútbol}

En los últimos años se han desarrollado múltiples trabajos que permiten establecer algunas de las condiciones fisiológicas a las que son sometidos los futbolistas durante un partido de fútbol; algunas de estas corresponden a estimaciones pues, por las mismas características del juego, las normas de reglamentación y protección del deportista, no es posible hacer cuantificaciones directas, menos aun cuando los partidos adquieren un compromiso de alto nivel como lo son las ligas o torneos profesionales. A continuación describiremos algunos de los hallazgos que desde el punto de vista fisiológico se han estudiado en la caracterización del futbolista.

\section{Gasto calórico y energético}

El gasto calórico (definido como la cantidad de calorías consumidas en un periodo), que para este caso es un partido de fútbol, depende 
de múltiples condiciones entre las que se encuentran el nivel de exigencia deportiva, la preparación física del futbolista, la posición de juego o el grado de compromiso durante la competencia. A pesar de lo anterior y de combinar diferentes técnicas de cuantificación, los valores han sido similares en cuanto a la cantidad de calorías consumidas.

Utilizando la frecuencia cardiaca durante el juego, se ha estimado que el consumo calórico está alrededor de las 1200 a $1500 \mathrm{Kcal}$ (Mohr, Krustrup y Bangsbo, 2005; Osgnach, Poser, Bernardini, Rinaldo y Di Prampero, 2010), esto a pesar de la limitación de utilizar pulsómetros (monitores de frecuencia cardiaca) durante partidos oficiales (Esposito et ál., 2004). Por otro lado, mediante cuantificación directa de consumo de oxígeno, el gasto calórico de deportistas profesionales se ha calculado entre las 1519 a 1772 Kcal (Stolen, Chamari, Castagna y Wisloff, 2005). A partir de lo anterior y en razón del peso corporal de los deportistas, el gasto de energía derivado del consumo calórico es de aproximadamente unos $61,12 \pm 6,57 \mathrm{KJ}^{\mathrm{kg}}{ }^{-1}$, donde las actividades de alta intensidad $\left(>20 \mathrm{~W} \cdot \mathrm{kg}^{-1}\right)$ representan cerca del $26 \%$ del tiempo de juego y corresponden aproximadamente al $42 \%$ del gasto energético total (Osgnach et ál., 2010).

El consumo calórico y por ende el energético son condiciones para tener en cuenta durante la preparación competitiva del deportista, ya que de la intervención nutricional previa y de los diversos métodos de recuperación nutricional postcompetitiva dependerá el contar con las reservas necesarias para el adecuado desempeño.

\section{Distancia recorrida}

Diferentes estudios han permitido establecer que durante un partido de 90 minutos es posible recorrer una distancia de hasta 12 kilómetros (Hoff y Helgerud, 2004), sin que obviamente se haga de forma continua, ya que un sprint o aceleración ocurre en promedio cada 90 segundos, y cada uno de estos dura entre 2 y 4 segundos, constituyendo del 1 al $11 \%$ de la distancia total recorrida. 
Tales valores varían ampliamente por posición de juego, y en general los defensas laterales pueden tener hasta 2,5 veces más sprint que los defensas centrales, mientras que los mediocampistas y los atacantes tienen aproximadamente 1,6 a 1,7 veces más sprint que los defensas centrales (Stolen et ál., 2005).

Comparado con las últimas décadas, múltiples y muy recientes trabajos han establecido que el fútbol actual es mucho más exigente físicamente y que las distancias que recorren los futbolistas profesionales son mayores; un estudio realizado por medio de análisis de video en 399 jugadores profesionales italianos demostró que en promedio ellos recorren durante un partido $10950 \pm 1044$ metros (Osgnach et ál., 2010), datos que no difieren mucho del promedio europeo por deportista, el cual está entre los 10 a $12 \mathrm{~km}$ por partido, sin grandes diferencias entre las posiciones (excepto por los arqueros que solo recorren alrededor de $4 \mathrm{~km}$ ) (Stolen et ál., 2005).

El hecho de que un futbolista cubra una distancia de hasta $12 \mathrm{~km}$ en 90 minutos es un fuerte indicador de la alta resistencia aeróbica que estos deportistas requieren en la actualidad.

\section{Exigencias aeróbicas en el futbolista}

El entrenamiento aeróbico es un componente importante en la preparación física del futbolista; prueba de ello son los estudios que demuestran la relación existente entre la potencia aeróbica, expresada en valores de consumo máximo de oxígeno $\left(\mathrm{VO}_{2}\right)$, y otras variables como el nivel competitivo, la calidad del juego, la distancia cubierta durante el juego, el tiempo en actividades de alta intensidad, el número de sprints y el número de toques del balón durante el juego (Impellizzeri, Rampinini y Marcora, 2005). Mediciones directas de consumo de oxígeno durante un partido son difíciles, pero algunas estimaciones permiten encontrar consumos promedio durante el juego entre los 45 a $52 \mathrm{ml} / \mathrm{kg} / \mathrm{min}$, correspondientes a consumos máximos de entre 60 a $70 \mathrm{ml} / \mathrm{kg} / \mathrm{min}$ (Stolen et ál., 2005). Cuando los futbolistas han sido sometidos a pruebas de laboratorio para la determinación del $\mathrm{VO}_{2}$ máximo, estos muestran valores oscilantes 
entre los 50 a $75 \mathrm{ml} / \mathrm{kg} / \mathrm{min}$ (los arqueros oscilan entre 50 a $55 \mathrm{ml} /$ $\mathrm{kg} / \mathrm{min}$ ); sin embargo, este conjunto de valores son muy superiores a los reportados por los futbolistas de la década de los ochentas (Stolen et ál., 2005).

Algunos datos cercanos a la realidad en nuestro medio muestran que el futbolista centro y suramericano tiene valores menores de $\mathrm{VO}_{2}$ : en Costa Rica un estudio con 219 jugadores mostró un $\mathrm{VO}_{2}$ max. de $57,71 \pm 8,8 \mathrm{mlO}_{2} / \mathrm{kg} / \mathrm{min}$ (Sánchez y Salas, 2009) y en Chile 40 futbolistas profesionales arrojaron un $\mathrm{VO}_{2}$ max. de 56,2 $\pm 5,7$ $\mathrm{mlO}_{2} / \mathrm{kg} / \mathrm{min}$ (Urzua, Von Oetinger y Cancino, 2009); en Colombia acceder a los resultados de mediciones directas no es fácil y muchos de los datos con los que se cuentan corresponden a mediciones indirectas.

Obviamente, existen muchas condiciones que hacen que el consumo de oxígeno varíe, entre ellos la temporada, pero también el nivel deportivo, la altura, las condiciones de evaluación, entre otras; sin embargo, lo que está claro es que los equipos de mejor clasificación mundial tienen mejores valores de $\mathrm{VO}_{2}$ que los peor ubicados en el ranking (Stolen et ál., 2005), siendo el $\mathrm{VO}_{2}$ un excelente predictor de rendimiento.

Por otro lado, no solo el consumo de oxígeno es importante, sino también el momento en el que ellos hacen la transición de trabajo aeróbico-anaeróbico (umbral anaeróbico, UA), en términos de porcentaje del consumo máximo de oxígeno. EI UA calculado en futbolistas profesionales adultos está entre el $76,6 \%$ al $90,3 \%$ de la frecuencia cardiaca máxima (Stolen et ál., 2005), sin que haya mayores reportes del UA en estudios previamente realizados, todo esto a pesar de la importancia que este tiene para la planificación del entrenamiento.

Por último, y también asociado a las exigencias aeróbicas en el fútbol, está la capacidad de cada uno de los deportistas para realizar el mismo esfuerzo con el menor gasto energético y la menor demanda fisiológica, algo que comúnmente se conoce como economía de carrera, la cual entre futbolistas profesionales y aficionados, 
según estudios previos, puede llegar a ser del $20 \%$, lo que en términos prácticos constituye cerca de $5 \mathrm{ml} / \mathrm{kg} / \mathrm{min}$ menos de consumo de oxígeno en un esfuerzo, o casi diez latidos menos ante un mismo esfuerzo, o cerca de 1500 metros menos por juego (Stolen et ál., 2005).

\section{Exigencias anaeróbicas en el futbolista}

Durante sus acciones de juego, los futbolistas se ven obligados a ejecutar constantemente actividades de alta intensidad y corta duración, un ejemplo de ello son las carreras cortas (sprint). La gran mayoría de los sprints son distancias entre 10 y $30 \mathrm{~m}$ (donde el $96 \%$ son menores a $30 \mathrm{~m}$ y $49 \%$ son menores a $10 \mathrm{~m}$ ) (Stolen et ál., 2005). Por tanto, los atletas tienen la obligación de entrenar y desarrollar las capacidades necesarias para afrontar estos esfuerzos de corta duración, de forma tal que para algunos autores la velocidad desarrollada en carreras de 10 o menos metros son representativas del componente anaeróbico en futbolistas (Chelly et ál., 2010a).

La evidencia ha demostrado que existe una adecuada relación entre el desarrollo de fuerza máxima a través de una repetición máxima (1RM) y la capacidad de realizar actividades de alta intensidad, como saltos de altura y carreras de 10 y $30 \mathrm{~m}$, respectivamente (Hoff y Helgerud, 2004); de allí que la fuerza máxima es la clave fundamental para el desarrollo de la potencia muscular, encontrándose una relación muy estrecha entre esta y la velocidad en deportes como el fútbol (Stolen et ál., 2005). Por otro lado, relaciones como la fuerza isométrica máxima, las características de curva tiempofuerza, la frecuencia de pedaleo en pruebas de bicicleta, fortalecen la importancia de la fuerza explosiva en deportes con exigencias anaeróbicas intensas como el fútbol (Gissis et ál., 2006).

La potencia y la capacidad anaeróbica pueden evaluarse de una forma efectiva a través de pruebas estandarizadas como los test de saltos (Bosco, 1994), o pruebas de potencia en bicicleta durante tiempos cortos como los 30 segundos (Inbar, Bar-Or, y Skinner, 1996), 
los cuales en fútbol han demostrado correlaciones estrechas y beneficios cuando se mejoran los resultados (Chelly et ál., 2010b).

\section{Efectos y demandas psicológicas en el fútbol}

La psicología deportiva es el aspecto más fascinante de la práctica deportiva, ya que es de vital importancia y de aplicación para entrenadores, deportistas, directivos y espectadores amantes del fútbol. Algunos autores la definen como "una ciencia consistente para aplicar los principios de la psicología en el deporte y en la actividad física, con el único fin de mejorar el rendimiento del deportista en la competencia" (Cox, 2007), pero en realidad la verdadera psicología del deporte es aquella que, además de ayudar a mejorar el rendimiento deportivo de los atletas, contribuye al enriquecimiento humano de todo un equipo de trabajo, en este caso en el fútbol.

Desde otra perspectiva, Roffé (2009) retoma la definición de psicología deportiva que hace García Ucha en 2007 en el marco del consenso de una amplia comunidad científica especialista en psicología del deporte y de la actividad física, donde se define como "la ciencia que estudia las regularidades deportivas de la actividad deportiva". En la actualidad la psicología deportiva se extiende a otros ámbitos comprometidos en el deporte, tales como el arbitraje, los entrenadores, los aficionados al deporte, etc. En este sentido y atendiendo a su objeto de estudio, es tanto una rama de la psicología como una ciencia aplicada al deporte.

Es importante destacar que los comportamientos y conductas de los deportistas en condiciones de entrenamiento y de competencia son observables; dichos comportamientos son producto de los aspectos cognitivos y mentales que mantienen en los atletas diferentes manifestaciones conductuales.

Para Roffé (2009), la psicología del deporte y de la actividad física constituye una especialidad que tiene por objeto el estudio científico 
de la subjetividad de las personas que participan en la práctica del deporte, tanto para la optimización del rendimiento como en el desarrollo personal del deportista, llevando a la investigación, a la enseñanza y a la práctica de los conocimientos específicos alcanzados por esta disciplina científica.

En esta línea, Dosil (2007) determina que definir el fútbol es un factor útil para enmarcarlo deportiva y psicológicamente; citando a Riera (1989), afirma que el fútbol es un juego con oponentes, en donde cada equipo debe coordinar sus acciones para mover, conservar y recapturar el balón, así como para poder desplazarlo a la zona de marca y anotar un gol. De igual manera, Dosil también afirma que el fútbol es una lucha por el dominio territorial dentro de un marco de reglas que incluyen aspectos estratégicos y técnicos, entre los que se destaca la anticipación.

\section{Características psicológicas en el fútbol}

Dosil sugiere una caracterización psicológica del fútbol atendiendo a cuatro elementos importantes:

1. Conocer cómo interactúan el conocimiento de base, las habilidades técnicas y las situaciones de juego.

2. Analizar el tipo de procesos cognitivos implicados en el fútbol.

3. Diferenciar entre "conocer" sobre el fútbol y ser "competente" en el fútbol.

4. Encontrar las posibles aplicaciones psicopedagógicas.

Los aspectos mencionados son las habilidades que deben tener un entrenador y su equipo de trabajo ante el comportamiento de sus futbolistas en los entrenamientos y en la competencia. De forma tal que el fútbol es su proceso de formación con variantes de "ensayo-error", debido a que los futbolistas se van probando en diferentes situaciones. 
Llames manifiesta cuatro características diferenciales, las cuales son totalmente entrenables y que deben combinarse entre sí:

1. Control y manejo de la activación.

2. Adecuada focalización de la atención.

3. Capacidad de afrontamiento.

4. Nivel óptimo de autoconfianza.

Estas características, junto con las físicas, fisiológicas, técnicas y tácticas, pueden proporcionar altas posibilidades de progreso en la práctica y en el rendimiento del fútbol.

Ahora bien, en la caracterización psicológica de futbolistas universitarios que entrenan a 2600 metros de altitud, es necesario determinar ciertas cualidades psicológicas especiales, teniendo como referente de partida las características propias de la competencia, las emociones y los sentimientos, los cuales generan diferentes tipos de comportamientos (totalmente observables) en cada uno de los futbolistas. A partir de esta consigna, el fútbol desde el punto de vista psicológico puede observarse desde estas perspectivas:

- La importancia deportiva: la parte económica y social que representa el campeonato y/o el partido por disputar.

- La posición del jugador dentro del campo de juego.

- Los momentos del juego: gol a favor, gol en contra.

- Los agentes externos que componen el ambiente del juego y del deportista: la familia, el público, el entrenador, el árbitro, el equipo adversario, etc.

García (2004), en su estudio sobre los aspectos psicológicos en el fútbol a través del entrenamiento integrado, destaca que el componente psicológico no se entrena, se hace, así como se desarrolla de forma aislada de las características del juego. También bajo esta perspectiva, el autor destaca la importancia del proceso de entrenamiento en los deportes de conjunto, denominándolo entrenamiento integrado o globalizado. 
Ahora bien, desde otro punto de vista ya más centrado en el juego como tal, durante el desarrollo de un partido de fútbol los jugadores experimentan diferentes comportamientos, muy ligados a su importancia (clasificación a la siguiente ronda, mantener la casilla en la categoría élite, la final del campeonato, premios económicos, becas, descuentos en la matrícula de la universidad, entre otros). La necesidad de obtener un resultado positivo, y por ende la angustia por no perder, también genera presiones psicológicas y comportamientos propios en sus practicantes, en este caso jugadores universitarios.

En la apreciación de Núñez (2011) existen diferentes situaciones durante un partido de fútbol las cuales van a provocar en los jugadores una serie de pensamientos, a su vez estos pensamientos generarán diferentes sentimientos y emociones, lo que lleva a condiciones de alegría, impotencia y/o frustración. De la misma manera, Gálvez y Paredes (2007) cuantifican los aspectos comportamentales de los jugadores durante un partido de fútbol. Ellos determinan que este deporte se relaciona con el esfuerzo individual del jugador y de la suma del equipo (cooperación) contra el esfuerzo de un equipo adversario, bien de forma individual o colectiva (oposición). Lo que saca a relucir y determinar el éxito o fracaso del o de los deportistas.

Dichos autores plantean aquellas características psicológicas que tiene o posee el jugador de fútbol:

- La personalidad del jugador. Weinberg y Gould (1996) la definen como "conjunto de características, o combinación de las mismas, que hacen que una persona sea única e independiente". Este aspecto está ligado al nivel de experiencia del jugador para afrontar los partidos independientemente de la importancia del mismo.

- La motivación. Sánchez y Zanger (2007) la definen como ese factor que hace mover al deportista sin pretender nada a cambio diferente a su placer propio. Este factor es el que hace que el deportista se esfuerce al máximo, consiguiendo solo su propio bienestar y placer. 
- La autoconfianza. Se define como el juicio o evaluación que hace una persona de sí misma en cuanto al valor personal se refiere. Aspecto que desarrolla un jugador de fútbol, según la posición dentro del campo de juego y su respectiva función para cumplir con los objetivos planteados a nivel técnico, táctico y estratégico.

- La concentración. Sánchez y Zanger (2007) la definen como la focalización de la atención en una tarea que se desarrolla en el presente, no existe ni el pasado ni el futuro, el deportista está en el presente y esa es su única realidad. Un solo momento de desconcentración puede generar fallas en la función del jugador dentro del campo de juego y por ende incidir en el resultado del mismo.

Los factores anteriormente mencionados se presentan con mayor o menor grado en cada uno de los jugadores de fútbol a la hora de ser agentes activos de un partido oficial de competencia a nivel nacional y/o internacional.

Tanto el compromiso fisiológico como los aspectos antropométricos, biomédicos y psicológicos de los futbolistas universitarios se han analizado por parte del Grupo de investigación en ciencias aplicadas al ejercicio físico, el deporte y la salud (GICAEDS), y una gráfica esquemática de los aspectos por evaluar en dicha investigación se encuentra en la figura 1.

Figura 1. Representación esquemática de la evaluación de futbolistas universitarios, que se ha desarrollado en la Facultad de Cultura Física, Deporte y Recreación

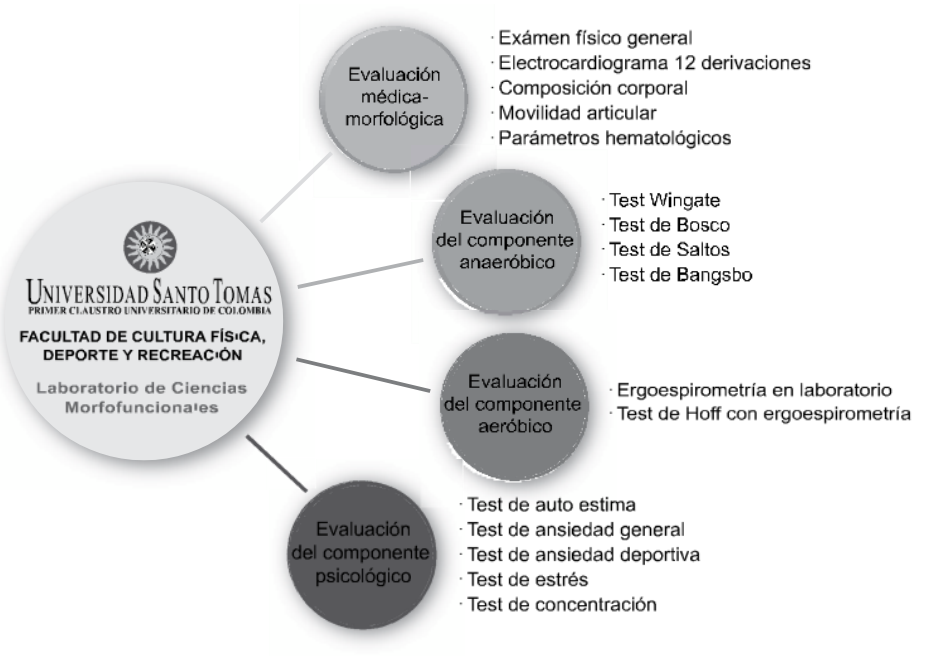




\section{Conclusión}

El futbolista moderno es un atleta con altas condiciones físicas y psicológicas; requiere la suficiente capacidad aeróbica y la economía de carrera para mantener durante 90 minutos desplazamientos multidireccionales continuos a diferentes intensidades, necesita la fuerza explosiva y la potencia anaeróbica para cortos cambios de dirección a máxima velocidad; de otra parte, requiere de una fuerte personalidad que lo lleve a un óptimo estado de motivación, autoconfianza y concentración durante todo el entrenamiento y el juego. Todo esto sin contar con unas elevadas condiciones técnico-tácticas y una alta disposición de trabajo en equipo.

En razón de las múltiples variables para tener en cuenta, el rendimiento del jugador de fútbol es el resultado de un estado de integralidad, razón por la cual no puede estudiarse ni entrenarse solo desde un único punto de vista; se requiere, sin lugar a dudas, de la interdisciplinariedad en la evaluación y la intervención deportiva. Esta revisión evidencia las necesidades físicas y psicológicas del futbolista en la actualidad, lo cual establece los parámetros de evaluación tanto fisiológica como psicológica de los futbolistas universitarios que viven y entrenan a 2600 metros sobre el nivel del mar.

\section{Referencias}

Bosco, C. (1994). La valoración de la fuerza con el test de Bosco (1a․ ed.). Barcelona: Paidotribo.

Chelly, M.S., Cherif, N., Ben Amar, M., Hermassi, S., Fathloun, M., Bouhlel, E. et ál. (2010a). Relationships of peak leg power, 1-RM half back squat and leg muscle volume to $5-m$ sprint performance of junior soccer players. J Strength Cond Res, 24(1), 266-271.

Chelly, M.S., Ghenem, M.A., Abid, K., Hermassi, S., Tabka, Z. y Shephard, R. (2010b). Effects of in-Season Short-Term Plyometric training program on leg power, jump and sprint performance on soccer players. J Strength Cond Res, 24(10), 2670-2676. 
Cox, R. (2007). Psychologie du sport. Sciences et pratique du sport. De Boeck.

Dosil, J. (2007). El psicólogo del deporte. Asesoramiento e intervención. Síntesis.

Esposito, F., Impellizzeri, F.M., Margonato, V., Vanni, R., Pizzini, G. y Veicsteinas, A. (2004). Validity of heart rate as an indicator of aerobic demand during soccer activities in amateur soccer players. Eur J Appl Physiol, 93(1), 167-172. doi:10.1007/s00421004-1192-4. Retrieved from Springer Berlin / Heidelberg.

Gálvez, A. y Paredes, J. (2007). Aspectos psicológicos que influyen en el futbolista. En efdeportes revista digital. Retrieved from http://www.efdeportes.com/efd106/aspectos-psicologicos-queinfluyen-en-el-futbolista.htm

García, T. (2004). El trabajo de aspectos psicológicos en fútbol a través del entrenamiento integrado. Madrid (España): CV Ciencias del Deporte.

Gissis, I., Papadopoulos, C., Kalapotharakos, V., I, Sotiropoulos, A., Komsis, G. y Manolopoulos, E. (2006). Strength and speed characteristics of elite, subelite, and recreational young soccer players. Res Sports Med, 14(3), 205-214.

Hoff, J. y Helgerud, J. (2004). Endurance and Strength Training for Soccer Players. Sports Med, 34(3), 165-180.

Impellizzeri, F., Rampinini, E. y Marcora, S. (2005). Physiological assessment of aerobic training in soccer. J Sport Sci, 23(6), 583592. doi:10.1080/02640410400021278

Inbar, O., Bar-Or, O. y Skinner, J.S. (1996). The Wingate Anaerobic Test (1… ed.). Human Kinetics.

Metaxas, T.I., Koutlianos, N., Sendelides, T. y Mandroukas, A. (2009). Preseason physiological profile of soccer and basketball players in different divisions. J Strength Cond Res, 23(6), 1704-1713. 
Mohr, M., Krustrup, P. y Bangsbo, J. (2005). Fatigue in soccer: A brief review. J Sport Sci, 23(6), 593-599. doi:10.1080/02640410400021286

Núñez, A. (2011). Aspectos psicológicos y rendimiento deportivo. http://www.fcylf.es/noticias/colaboracion.asp?id=3

Osgnach, C., Poser, S., Bernardini, R., Rinaldo, R. y Di Prampero, P.E. (2010). Energy Cost and Metabolic Power in Elite Soccer: A New Match Analysis Approach. Med Sci Sports Exerc, 42(1), 170-178.

Reilly, T. y Gilbourne, D. (2003). Science and football: a review of applied research in the football codes. J Sport Sci, 21(9), 693705. doi:10.1080/0264041031000102105

Roffé, M. (2009). Evaluación psicodeportológica. 30 test psicométricos y proyectivos. Lugar Editorial.

Sánchez, A., \& Zanger, A. (2007). La préparation mentale du joueur de Torball de Brest. Université de Bretagne Occidentale.

Sánchez, B. y Salas, J. (2009). Determinación del consumo máximo de oxígeno del futbolista costarricense de primera división en pretemporada 2008. MHSalud, 6(2), 1-5.

Stolen, T., Chamari, K., Castagna, C. y Wisloff, U. (2005). Physiology of Soccer, An Update. Sports Med, 35(6), 501-536.

Svensson, M. y Drust, B. (2005). Testing soccer players. J Sport Sci, 23 (6), 601-618. doi:10.1080/02640410400021294

Urzua, R., Von Oetinger, A. y Cancino, J. (2009). Potencia aeróbica máxima, fuerza explosiva del miembro inferior y peak de torque isocinético en futbolistas chilenos profesionales y universitarios. Cronos, VIII(14), 42-52.

Weinberg, R. y Gould, D. (1996). Fundamentos de psicología del deporte y del ejericio físico (3ª ed.). Madrid: Panamericana. 